\title{
Opportunities of Telemedicine for Orthopedics and Trauma Surgery
}

\author{
Michael Nerlich ${ }^{1 *}$, Tanja Herbst ${ }^{1}$, Antonio Ernstberger ${ }^{1}$, Markus Blaetzinger $^{2}$ \\ ${ }^{1}$ Department of Trauma Surgery, University Hospital Regensburg, Regensburg, Germany \\ ${ }^{2} \mathrm{AUC}$ - Akademie der Unfallchirurgie GmbH Munich, Munich, Germany
}

Corresponding Author: Michael Nerlich, MD, Professor, Department of Trauma Surgery, University Hospital

Regensburg, Regensburg, Germany. Tel: 0941/944-6805, Email: michael.nerlich@ukr.de

Received February 4, 2019; Accepted April 25, 2019; Online Published June 6, 2019

\begin{abstract}
Today telemedicine, or eHealth, is an important tool in everyday medical life. In all areas, from the preclinical to aftercare, significant improvements in communication structures have been seen in various eHealth options, which have had significant, positive effects on the quality of patient care in orthopedics and trauma surgery. Initially, there were several isolated solutions and many small individual projects; however, there was a lack of interdisciplinary and comprehensive systems in all healthcare fields. The German Trauma Society (DGU) together with the AUC GmbH took the lead and paved the way for a modulated, comprehensive, interface-compatible teleradiology system. Nevertheless, there are still deficits across all sectors which, in the future, will need to be optimized by eHealth methods and systems. Overall, there is an effort towards patient-centered solutions (patient empowerment). In Germany, telemedicine has gradually gained acceptance in various sectors and is being used nationwide. Telemedicine has proven itself, especially in trauma networks.

Keywords: Telemedicine, Teleradiology, eHealth, Orthopaedics, Trauma Surgery, Patient Care, Patient Participation
\end{abstract}

Citation: Nerlich M, Herbst T, Ernstberger A, Blaetzinger M. Opportunities of telemedicine for orthopedics and trauma surgery. Int J Travel Med Glob Health. 2019;7(3):72-78. doi:10.15171/ijtmgh.2019.17.

\section{Introduction}

The Development and Necessity of Telemedicine from Preclinical to Post-treatment Care

The awareness of fundamental communication issues initially led to the development of telemedicine strategies and solutions. Today it is well known that communication in any form is generally affected by the information and perception biases of the sender and receiver. The sender's message is usually transmitted incompletely and may be received with a considerably different interpretation then intended. Particularly in medicine, efficient, immediate communication among preclinical, clinical, and post-treatment care is essential and vital. Thus, a great effort is being made to transmit information as effectively as possible. Telemedicine solutions ideally serve to deliver time-saving, structured, focused, and thorough information between sender and receiver.

Over the past years, telemedicine has initiated a change in healthcare structures and medical sectors. Hospital processes are increasingly based on electronic communication systems, which facilitate various medical services. ${ }^{1}$ The development and implementation of eHealth systems is also achieved rapidly, because junior staff members in particular are accustomed to IT and information exchange through social media, and thus accept it without reservations.

A lot of progress has been made in telemedicine over the past years, especially in orthopedics and trauma surgery. More and more of the communication between preclinical and clinical care uses electronic assistance, and the transmission of radiologic images between trauma network hospitals has been implemented nationwide. In orthopedics and trauma surgery, the catchphrase "patient empowerment" describes the growing number of competent patients who wish to be involved in the diagnostic process as well as in treatment decision making. Online chats or video calls with the responsible physician are currently less appealing for patients; however, web-based organizational tools like online appointment schedulers are well-received. ${ }^{2}$ The first steps in achieving a wider acceptance and increasing the implementation of telemedicine solutions in follow-up treatment have been made, focusing particularly on resident doctors.

\section{Preclinical Care}

In the last few years, a deficiency in preclinical care has become apparent throughout Germany. Various structural changes have resulted in a shortage of doctors in rural areas (i.e. demographic development, medical advances, decrease of

Copyright $(0) 2019$ The Author(s). This is an open-access article distributed under the terms of the Creative Commons Attribution License (http:// creativecommons.org/licenses/by/4.0), which permits unrestricted use, distribution, and reproduction in any medium, provided the original work is properly cited. 
medical practices in the country, lack of general practitioners, work overload of hospital doctors, closure of hospitals etc). Access to general practitioners and specialists is influenced by an increasingly unequal regional distribution. At the same time, due to the lack of doctors in rural areas, fewer physicians are available to operate as qualified emergency doctors. ${ }^{3}$ This human resource deficit results in inadequate preclinical patient care. The widely mandatory time limit of 12 minutes between receiving an emergency call and arriving at the accident site is not feasible. Consequences of the shortage of emergency doctors are most notable in sparsely populated regions of the country. A delayed arrival of the emergency doctor can have significant effects on a patient's outcome. Despite the structural changes, high-quality, efficient patient care must be ensured. For almost 30 years, extensive interdisciplinary research on various preclinical topics has been conducted throughout Germany, in an attempt to generate new ideas for combatting the physician shortage in rural areas and to counteract associated problems using electronic solutions. ${ }^{4}$

\section{The Onset of Telemedicine in Regensburg: NOAH}

Telematic solutions can close the gaps in preclinical care, particularly those in sparsely populated regions. Telemedicine is the appropriate instrument for overcoming distances in emergency care, because it enables the speedy transmission of important information from the accident location to the hospital. Particularly in severe, life-threatening situations, a quick electronic exchange of information is essential. Stroke and cardiac arrest networks are already considerably advanced; nonetheless, traumatology treats severely injured patients and depends on optimum preclinical structures as well. These structures are supported by feasible telemedicine systems, facilitating optimal care for patients from the accident location to hospital admission.

The first attempts to enhance communication between preclinical and clinical care through telematic solutions at Regensburg University Hospital were undertaken twenty years ago. Specific focus was placed on the structured, electronic transmission of data from the emergency doctor to the hospital. The "Notfall-Organisations-und Arbeitshilfe" (NOAH) Project enabled the collection and concise, structured transmission of data in a matter of seconds to the rescue coordination center or hospital. ${ }^{5} \mathrm{~A}$ field trial verified the technical feasibility as well as the conceptual design of the NOAH system regarding loss-free data transmission. Moreover, utilization of the NOAH system led to an over twenty-minute time gain in the hospital setting. The tests also showed a considerable improvement not only in the quantity, but especially in the quality of transmitted information. ${ }^{6}$

The NOAH Project in Regensburg was the first to prove the feasibility of using this type of communication system for emergency services. This groundbreaking project was subsequently adopted and enhanced, leading to the development of "NIDA", an assistant for documenting emergency situation information. NIDA is designed similarly to a tablet and specifically for mobile use in preclinical care. The system documents information collected during an emergency situation or patient transport, e.g., patient information, vital signs, logistical data and medical procedures, and transmits it to the hospital. In 2014 the Project "Telematik II" by the Bavarian Red Cross and Bavarian Ministry of Interior included the nationwide implementation of the NIDA-Pad in all public ambulance services. ${ }^{7}$ These devices are now indispensable in preclinical care, saving both time and lives. ${ }^{8}$ The NIDA-Pad accelerates data flow and structures preclinical care, allowing processes to be simplified and communication errors to be minimized. Moreover, an exact and complete documentation is possible, in accordance with the Bavarian rescue service's legal requirements for quality management and scientific analysis. ${ }^{9}$

\section{Establishing the Tele-Emergency Medical Service in Preclinical Care}

Today, similar preclinical concepts are used widely, the feasibility and basic functions having already been tested in the NOAH Project. For example, the Aachen tele-emergency medical service ${ }^{10}$ was established, enabling immediate medical emergency competence. This service provides support to preclinical care rescue services and during patient transportation. Their communication unit provides secure, reliable voice and data communication through mobile communication (audio, video, vital signs, and photos). The transmission of relevant information allows for high quality emergency care, increases the efficient use of the rescue services resources, and improves the flow of information along the whole rescue chain.

Currently, the Bavarian tele-emergency doctor service, originating from the Straubing district, is revolutionizing modern rescue service care. ${ }^{11}$ This e-health model focuses on supporting rescue services at the accident location so as to reduce the time a patient spends without treatment until the arrival of an emergency doctor. Through modern technology, the tele-emergency doctor has access to images and medical data transmitted in real time from the location by the rescue service team, allowing immediate advice via virtual communication. Responsibility for the patient is then assumed by the emergency doctor upon arrival on site.

Both previously mentioned projects pay great attention to data security so as to provide optimum protection of personal patient data in accordance with the latest standards. Moreover, patients benefit greatly from these systems, as the therapyfree interval is shortened via telemedicine, facilitating on-site emergency competence.

\section{Inter- and Intra-clinical Communication: Telecooperation TNW $^{\circledR} /$ TKmed $^{\circledR}$}

In addition to the progress in telemedicine for preclinical care, modifications in inter- and intra-clinical communication are also essential to improving quality of care. Telemedicine is already well established in the treatment of heart attack and stroke patients. However, orthopedics and trauma surgery also benefit from an efficient teleradiology network which connects trauma centers locally, regionally, and nationwide.

In 2009, the East Bavarian Trauma Network (TNO) initially considered the transmission of images inside the network of 25 certified trauma centers of various levels. The rural 
eastern Bavarian region is sparsely populated in comparison with metropolitan areas, entailing longer distances between clinics. ${ }^{12}$ TNO had merely one, central maximum care provider - the Regensburg University Hospital (UKR). There were insular solutions on the telemedicine market; however, these were exclusively manufacturer-bound. The hospitals worked with various internal communication systems, although these were hardly compatible with the teleradiology systems of other manufacturers. Therefore, the Orthopedics and Trauma Surgery Department of Regensburg University Hospital was keen on implementing functional, interface-compatible, and vendor-independent electronic telecommunication in the East Bavarian Trauma Network. From 2010 until 2012, a promising new prototype named Exdicomed was developed to meet this specific, regional need, and its implementation was funded through the "Leitprojekte Medizintechnik" program (Lead Projects in Medical Technique) of the Bavarian Ministry of Economy and Media, Energy, and Technology (StMWi) in conjunction with subsidies from the European Funds for Regional Development in Bavaria (EFRE). This innovative project, which could process common data formats (DICOM, JPG, PDF, etc), was designed to be flexible, safe, vendor-independent, and interface-compatible. Therefore, all interested hospitals and trauma networks could use it without technological restrictions. The TNO, consisting of 25 hospitals, functioned as a pilot network during the test phase. Throughout the two-year development phase in the UKR, this novel system received very positive feedback from the pilot network. Moreover, the positive effect on quality of care was notable. ${ }^{13}$

Based on this successful, functional, and innovative Bavarian pilot project, the DGU commissioned the AUC to develop $\mathrm{TKmed}^{\circledR}$, a radiology data transmission system, in $2012 .{ }^{14}$ The goal was to integrate this system nationwide in the medical sector as well as the DGU Trauma Network, thus increasing patient safety through telecommunications and improving the outcome of seriously injured patients. ${ }^{15}$ The DGU's requirement of functioning teleradiology in the Whitebook Medical Care of the Severely Injured (Figure 1) also contributed to the nationwide implementation of telemedicine in German trauma networks. Today, more than 500 recipients from all levels of care, including numerous university and BG-hospitals (statutory accident insurance hospitals), are connected with the telecooperation $\mathrm{TNW}^{\circledR} / \mathrm{TKmed}^{\circledR}{ }^{16}$ Initially developed as Exdicomed by the Regensburg University Hospital between 2010 and 2012, the AUC are now responsible for providing this interface compatible and data protection compliant teleradiology system on behalf of the DGU. ${ }^{17}$

From the start, TKmed $^{\circledR}$ pursued its own development philosophy. While most providers focused exclusively on the technique of a telecooperation system, TKmed ${ }^{\circledR}$ always kept an eye on the user as a decisive component, enhancing clinical routine with technology. ${ }^{18}$ Hence, the TKmed system is valued as a network created by physicians for physicians. Trauma centers of all levels rely on fast and functioning intercommunication, both to save valuable time in polytrauma care and to impart knowledge (second opinion) to smaller, peripheral hospitals, saving time and possibly even preventing

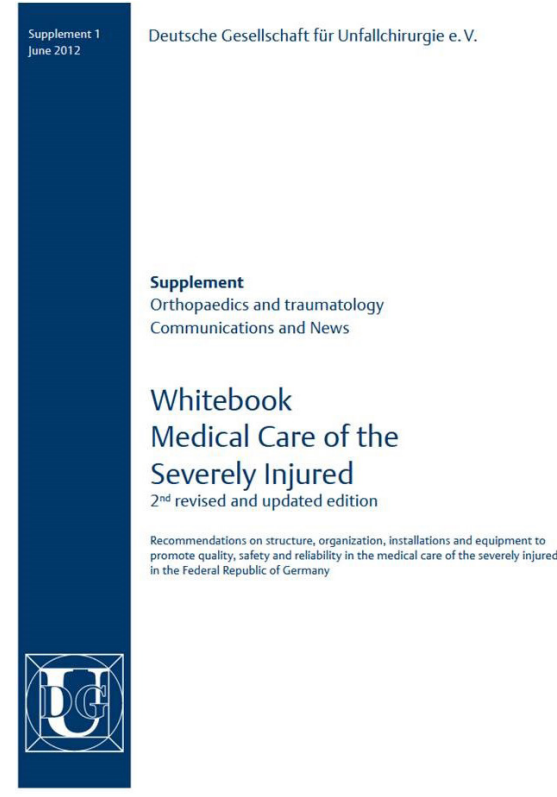

Figure 1. Cover Whitebook ${ }^{\mathrm{DGU}}$. $\mathrm{CAUC} \mathrm{GmbH} / \mathrm{DGU}$

patient transfers, improving quality of care considerably.

\section{Emergency Transfers}

The first certified DGU Trauma Network (TNW) was initiated in Germany in 2008. Each network is a regional collaboration of 10 to 30 trauma centers with one central maximum care provider (usually a university hospital). The DGU Whitebook Medical Care of the Severely Injured recommended that the networks should establish a secure and stable communication platform simultaneously, so as to transfer patient data and radiological images in compliance with data protection. ${ }^{19}$ The aim of implementing digital communication in trauma networks was to increase the medical process quality, outcomes in emergency care and patient safety, and the workflow in hospitals and clinical practices. Furthermore, the regional differences in quality of care for trauma patients were to be eliminated, resulting in a nationwide, high standard of treatment for seriously injured patients.

The teleradiological connection of hospitals in the trauma networks has been well established, particularly for emergency transfers. Frequently, a seriously injured patient receives first medical treatment in a local or regional trauma center and is then transferred to a maximum care hospital. Previously radiological images were burned on a CD in the local trauma center and handed to the patient transport. This meant that the images could be imported and viewed on a computer only after arrival, losing valuable minutes in patient treatment. Today, with the support of teleradiology, images are transmitted in seconds to the maximum care hospital. They can be observed there before the patient's admission, and appropriate measures for the patient's treatment can be taken.

\section{Second Opinion}

Today, seeking a second medical opinion is an essential component of patient autonomy, often because of uncertainty 


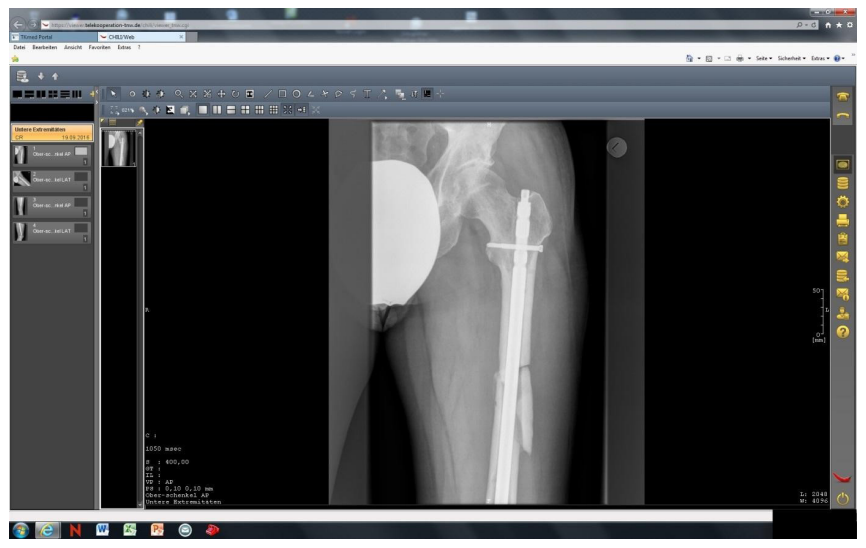

Figure 2. Teleradiological transmission via TKmed®.

pertaining to the appropriateness and consequences of medical advice. Generally, doctors will recommend seeking a colleague's opinion if a patient has concerns. This either leads to further options or the elimination of concerns if both medical statements are identical. Some specialists offer specific teleconsultation hours, so that patients do not have to come in person, which is advantageous for sparsely populated and less developed regions (Figure 2).

Patients benefit from a second opinion via $\mathrm{TKmed}^{\circledR}$, as it transfers expertise to small, peripheral hospitals in the trauma network, thus avoiding unnecessary, exhausting patient transfers or redundant examinations (radiation exposure). Images are sent to an expert at the maximum care provider (Figure 3), who assesses them and sends the results back to the doctor in charge at the inquiring hospital. Consequently, the competence of small hospitals is enhanced through the teleradiological second opinion.

\section{Referral and Patient Empowerment through TKmed- Direkt $^{\circledR}$}

In October 2015, TKmed ${ }^{\circledR}$ entered a new and innovative development stage with the availability of the TKmed ${ }^{\circledR}$-Direkt modul. ${ }^{20}$ This system allows the equal participation of patient and relatives in a digital communication solution, a patientcentered digital system. In sum, the development steps are as follows: technique-centered system (past) > physiciancentered system (present) > patient-centered system (goal, future).

Patients, established specialists, and rehabilitation facilities should be able to upload their own radiological images to the communication platform and send it to the respective recipient using any web-enabled computer. This would empower patient communication, enabling a treatment plan based on an equal partnership. Furthermore, direct and comprehensive communication enhances optimal patient safety as well as prompt treatment. With this system, the patient can save time and effort by transmitting images and documents directly online to the respective physician, avoiding waiting times, unnecessary travel, and redundant tests (radiation exposure). It also facilitates the hospital workflow, which is another benefit for patients. Simultaneously, healthcare costs are reduced, but quality of care is improved.

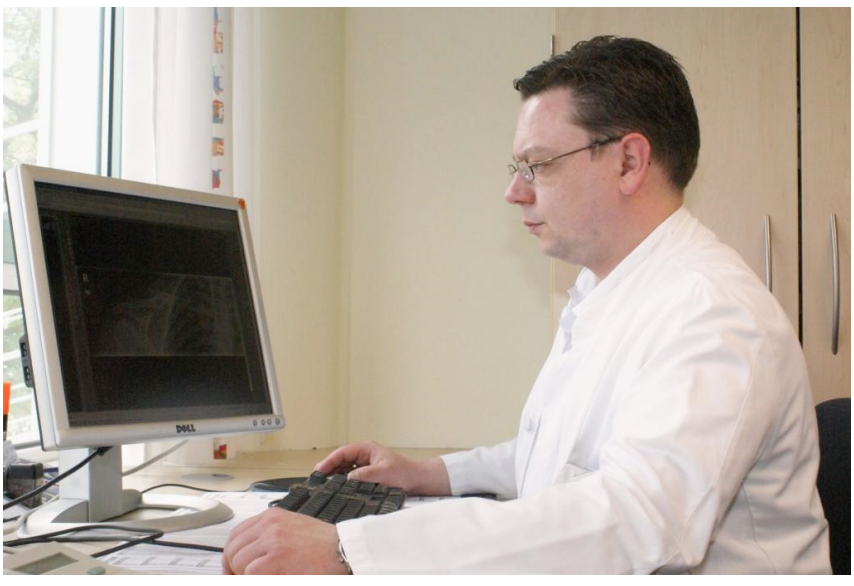

Figure 3. Second Opinion OUKR, A. Ernstberger

\section{The Quality of Telecommunication: TeleQualy}

Since December 2014, the project TeleQualy-I has been evaluating the teleradiology system TKmed $^{\circledR}$. This research project is a collaboration between the AUC and Regensburg University Hospital's Department of Trauma Surgery. To continually evaluate the system, a user survey is conducted, and the results are then scientifically analyzed. Because of continuous technical development, the evaluation not only covers technical issues, objective details, and transfer rates, but through TeleQualy the wishes and suggestions of users are also taken into consideration. This important feedback, based on the clinical user's experience, leads to useful and prompt technical modifications. Currently, a survey is being conducted on the utilization and value of radiology systems in the orthopedic and trauma surgery departments of university hospitals in Germany. ${ }^{21}$

In the future, the research project TeleQualy-II will evaluate the additional module TKmed ${ }^{\circledR}$-Direkt. The aim is to scientifically analyze the direct communication between inpatient facilities and the patient or an established specialist, focusing on patient safety and patient satisfaction. An indepth evaluation of this novel communication is important, as feedback from the users and scientific analysis will allow the system to be continually adapted and optimized.

\section{Post-treatment: Follow up Treatment, Rehabilitation, Resident Physicians}

The fundamental concept of telemedicine is to overcome long distances. Internationally, the possibilities of using digitization to transmit images was first identified and used in areas with inadequate medical care due to the lack of human resources. Therefore, the initial steps taken in eHealth at Regensburg University Hospital were developed for abroad. The idea was that the expertise of a German university hospital could be shared by video transmission with developing and emerging countries, which are often underserved in medical care. Since 1998, Regensburg University Hospital has been working on a project of the G8 countries. In 2002, the task force developed a recommendation for teleconsultations in clinical routine. ${ }^{22}$ This was the first step to developing general guidelines for telemedicine/teleconsultations. The researchers in Regensburg were very experienced in telemedicine 
and played a leading role in the International Society for Telemedicine \& eHealth (ISfTeH) right from the start. ${ }^{23}$ Their experiences with eHealth and telemedicine were essential for the restructuring of ISfTeH, changing individual membership to country membership and enabling worldwide recognition as an international influential umbrella company with a link to the World Health Organization. The objectives of this association were to disseminate knowledge and experience in telemedicine and eHealth across the globe and to facilitate access to recognized experts in this area. Through this cooperation, telemedicine and eHealth in Germany became generally accepted by the medical profession and could register the first increase in use.

As the first international endeavors of telemedicine were so successful, the idea to use it in Germany emerged quickly. Though the level of medical care seemed sufficient, on closer examination, deficiencies did emerge, such as poor connections to interregional hospitals (especially in regions like Eastern Bavaria) for older, immobile patients who have difficulty traveling long distances. Hence, diverse pre- and interclinical eHealth structures have been developing quickly.

In 2005, Eberl et al. published the results of a prospective, randomized study performed by the BG Hospital Bergmannsheil Bochum in the field of orthopedic and trauma surgery. This study analyzed post-treatment care through televisits of patients having undergone elbow arthroplasty in comparison to conventional follow-up care. There was no significant difference in outcomes between the groups, but the patients showed a high level of satisfaction with the handling of a televisit. ${ }^{24}$ Moreover, the time saved for both the patient (no travel required) and the physician was noticeable. In general, this study showed a subjective improvement in the patient's return to their home environment, shortened convalescence, and a rapid rehabilitation period. The use of televisits is feasible and would improve the doctor-patient partnership so that further utilization possibilities are plausible.

Regensburg University (ICT Regensburg) and the Aristotelian University of Thessaloniki (Medical School \& Lab of Medical Informatics) collaborated in 2004 on the project INTERLIFE to implement the already developed, innovative Home-Care-Systems. This system's goal was earlier hospital discharges with regular follow-up treatment at home, based on electronic communication. The main focus of the EU project was to transform the prototype of a wireless "Home Monitoring" (developed in the previous CHS project) into a product which could be marketed. The project included market research as well as contact with potential commercial providers and the target group. To complete the project, a study using patient participation to demonstrate the advantages of this system was performed. ${ }^{25}$

\section{Televisit, Homecare, and Video Consultations}

The shortage of physicians, particularly in rural regions, can be partially compensated with telemedicine projects. In these areas, opportunities offered by telemedicine have been well accepted and are gradually developing and expanding. A current and award-winning eHealth concept is the Teledoctor service. ${ }^{26}$ This model focuses mainly on general practitioner care. The physician pre-selects patients and delegates some tasks, such as collecting vital signs, to specifically trained telemedical assistants who assist patients onsite and transmit the data electronically to the clinical practice. The physician in the practice can access the transmitted data and, if necessary, connect via videoconference. The physician is provided with all medical information, simultaneously saving time and resources, and the patient still receives adequate care while at home. Some health insurance companies support this project, which has already won the Telematik-Award (in 2017) as a concept with a promising future. Trauma and orthopedic surgery could consider the use of this model for patients requiring outpatient treatment.

The possibility of conducting online video consultations according to all data protection guidelines is of particular interest to physicians. Online consultations can save time for both the patient and the physician, for example, for wound inspections or medical counselling. To bridge longer distances between patient and specialist, this can be an adequate solution in certain cases. A promising step in the direction of a wider acceptance of this form of telemedicine is the fact that since April 2017, video consultations are officially recognized and covered by insurances. The E-Health Act required the financial support of online consultations in July 2017. The National Association of Statutory Health Insurance Funds (GKV) and that of physicians (KBV) agreed on the necessary technical requirements for both the doctor's practice and the video service, paying specific attention to technical safety as well as data protection. The service provider must be certified, and end-to-end encrypted transmission is mandatory. ${ }^{27}$

In May 2018, the "Deutsche Aerzteblatt" (medical journal) reported on the relaxation of the ban of exclusive remote treatment. ${ }^{28}$ Furthermore, a large majority of the delegates of the 121st German Congress of Physicians (Deutscher Aerztetag) approved an amendment of the medical code of conduct. In the future, physicians may advise and treat specific patients using telemedicine without having had prior contact with them. In these cases, it is required that the patient be informed of both the advantages and the disadvantages of exclusive remote treatment, and the treatment must be both medically justifiable and performed with due diligence. ${ }^{29}$

Germany can strengthen its position internationally through these new regulations, as other countries have already integrated remote treatment in routine medical care. The new regulations provide doctors more flexibility in conducting treatments in the patient's best interest. Telemedicine does not replace personal patient contact, but it is a suitable method to overcome distances and avoid unnecessary time expenditures and efforts, especially for immobile patients.

\section{Rehabilitation and Follow-up Treatment}

Telemedicine networking is already well-advanced in some areas; however, patient treatment continues after hospital discharge. In particular, severely injured trauma patients have a long and arduous road to recovery. Followup treatment cannot be ignored by the telemedicine network. In orthopedics and trauma surgery, the electronic 
connection to rehabilitation facilities and discharged patients can still be improved. The main deficit in this field is the interinstitutional availability of treatment data. Due to a lack of both adequate IT solutions and process integration, the potential for the delivery of relevant treatment data is utilized neither between various forms of care (inpatient - outpatient - homecare treatment) nor inside the sector (acute hospitals - rehabilitation clinics, general physician - specialist). The professional responsible for the post-treatment still has to ask the patient for medical and treatment histories contact other institutions by telephone, or wait for previous findings to be sent.

\section{The Importance of Politics and Data Protection}

The importance of telemedicine as a modern element for an efficient healthcare is apparent for politicians. In 2014, Melanie Huml, the Minister for Social Affairs, stated that telemedicine plays an essential role in ensuring healthcare for all, especially in sparsely populated regions. She also noted that it can help close gaps in the healthcare system and make it more efficient. Telemedicine must be recognized as a regular benefit by health insurance. ${ }^{30}$

It should be noted that the acceptance and funding of telemedicine has increased greatly over recent years, both by the political front and by social insurance agencies, although there is still a deficit as to which costs are covered. As of yet, there is no explicit cost agreement for telemedical or teleradiological services, but only temporary solutions to sidestep this problem.

The financial support of eHealth and telemedicine projects by innovation programs and research initiatives of ministries is extremely important and enables research and development in this area. In addition to IT companies, medical institutes and university hospitals are key players in the development of new software, using their medical expertise to transfer theoretical concepts to clinical routines. In particular, research associations comprised of IT and medical personnel are the ones most capable of generating the best know-how and developing promising and user-oriented projects in this field. University hospitals depend on funding from the European Union or federal or local governments to conduct high quality eHealth research, as the demands on resources such as staff, software and hardware, structure and data protection are high. It is, therefore, gratifying to see that both federal and local governments support such projects with various initiatives. Only through continuous new and further developments and the implementation of ideas and possibilities in telemedicine can progress in the establishment of structures in Germany be possible. This will enable the use of telemedicine and eHealth in many sub-sectors of the healthcare system.

Patient data is a valuable resource of a very sensitive nature. Thus, many patients are anxious about how their personal data will be processed in eHealth systems. It is essential that any telematic solution be designed with the best possible technical and organizational measures, as data protection is of utmost importance. This can ensure that any unauthorized access or misuse by third parties is widely eliminated. The already established and prevailing systems process patient data in accordance with the legal requirements. The new EU General Data Protection Regulation (EU GDPR) that has been in effect since May $2018^{31}$ has the positive aspect of reinforcing the right to determine how personal information is handled. However, the modifications in data handling and the relevant and inevitable extra administrative burden limit some telemedical solutions, especially those for emergency situations.

\section{Conclusion and Perspectives}

Does the Field of Orthopedics and Trauma Surgery Take Full Advantage of Telemedicine?

Currently, eHealth and telemedicine play an indispensable role in the healthcare system, and its implementation in the various medical sectors will eventually take place. The positive factors for the clinical routine are evident: both time and costs can be saved, and the quality of patient care can be improved through efficient and structured communication. Today and in the future, cross-sectoral modular telematic solutions based on clinical practices and in line with data protection regulations are essential. Hospitals and clinical practices generate a lot of data daily. This data can efficiently facilitate research and future developments as long as data protection regulations are respected. Data has already been collected nationwide in registers and data banks, e.g., the trauma register of the German Society of Trauma Surgery. Regularities can be observed and extracted from the extensive data and allow for new insights for medical treatment. In the coming years, innovative ideas and opportunities will arise through the collaboration of these data banks, enhancing more effective and detailed results when the available data is analyzed. ${ }^{32}$

Various efforts have been made to constructively integrate telemedical systems in the routine care of orthopedic and trauma surgery patients. The opportunity provided by the Whitebook Medical Care of Severely Injured (DGU) ${ }^{19}$ was used to develop TKmed ${ }^{\circledR}$ and implement it as a functioning communication system for trauma networks. Nevertheless, the Department of Orthopedics and Trauma Surgery could learn a lot from other fields, specifically for follow-up treatment and communication between hospitals and acute rehabilitation facilities. EHealth supported methods are increasingly being used for injury prevention, for example, for sports or traffic accidents. The goal is to identify injury risks and patterns in advance, to analyze these, and to devise effective measures or products to avoid injuries with the help of telematic solutions.

The additional support from politics, medical societies and advisory telemedicine alliances is a good basis for further efforts in this area. This cooperation permits advancements in communication structures to improve patient care in orthopedics and trauma surgery.

\section{Authors' Contributions}

All Authors contributed equally to this study.

\section{Conflict of Interest Disclosures}

The authors declare that they have no conflicts of interest. 


\section{Ethical Approval}

Not applicable.

\section{Funding/Support}

None.

\section{References}

1. Neue Versorgungsformen im Gesundheitswesen? https://www.asuarbeitsmedizin.com/Archiv/ASU-Heftarchiv/article-767572-110576/ neue-versorgungsformen-im-gesundheitswesen-.html.

2. Holderried M, Schlipf M, Hoper A, Meier R, Stockle U, Kraus TM. [Chances and risks of telemedicine in orthopaedics and trauma surgery]. Z Orthop Unfall. 2018;156(1):68-77. doi:10.1055/s-0043-116941.

3. Droht ein Notstand bei der ärztlichen Versorgung von immer mehr alten und kranken Menschen? https://www.landkreistag.de/ themen/medizinische-versorgung.html?start=2

4. ÄrztlicheVersorgung im ländlichen Raum. https://www.bayerischesaerzteblatt.de/fileadmin/aerzteblatt/ausgaben/2008/09/einzelpdf/ BAB_0908_538_539.pdf.

5. Schachinger U, Kretschmer R, Neumann C, Nerlich M. NOAH. A mobile emergency care system. Notfall-Organisations- und Arbeitshilfe. Stud Health Technol Inform. 1999;64:85-92.

6. Schachinger $U$, Kretschmer R, Rockelein W, Neumann C, Maghsudi M, Nerlich M. NOAH--A mobile emergency care system. Eur J Med Res. 2000;5(1):13-18.

7. Telematik II. https://www.rettungsdienst.brk.de/abteilung/ landrettung/telematik-2.html.

8. Stadler T, Taheri-Sohi S. Datenschnittstelle Rettungsdienst und Notaufnahme: Mit Telematik die Patientenversorgung optimieren. Kma. 2018;23(9):52-54. doi:10.1055/s-0036-1595408.

9. NIDApad. https://www.meddv.de/de/11-produkte-und-loesungen/ einsatzdokumentation/17-nidapad

10. Telenotarzt website. https://www.telenotarzt.de/.

11. Wer ist Telenotarzt? Telenotarzt website. http://www.telenotarzt. bayern/was-ist-telenotarzt/.

12. Ernstberger A, Koller M, Zeman F, et al. A trauma network with centralized and local health care structures: Evaluating the effectiveness of the first certified Trauma Network of the German Society of Trauma Surgery. PLoS One. 2018;13(3):e0194292. doi:10.1371/journal.pone.0194292.

13. Ernstberger A, Leis A, Herbst T, Nerlich M. [Comprehensive medical care with telemedicine]. Zentralbl Chir. 2014;139(1):1-3.

14. TKmed website. http://tkmed.org/.

15. Ruchholtz S, Lefering R, Debus F, Mand C, Kuhne C, Siebert H. [TraumaregisterTraumaNetwork $\mathrm{DGU}(\mathrm{R})$ und TraumaRegister DGU(R). Success by cooperation and documentation]. Chirurg. 2013;84(9):730-738. doi:10.1007/s00104-013-2474-3.

16. TKmed®/TeleKooperation TNW®. https://www.auc-online.de/de/ telemedizin.html.

17. Staemmler M, Walz M, Weisser G, et al. Establishing end-to-end security in a nationwide network for telecooperation. Stud Health Technol Inform. 2012;180:512-516. doi:10.3233/978-1-61499101-4-512.

18. TKmed $^{\circledR}-$ Telekooperation für die einrichtungsübergreifende Versorgung. e-Health; 2014. https://www.chili-radiology.com/ fileadmin/user_upload/Publikationen_PDFs/2013-TelemedFuehrer-TKmed.pdf.

19. Whitebook Medical Care of the Severely Injured. $2^{\text {nd }}$ Ed. Berlin: German Society for Trauma Surgery; 2012. https://www.dgu-online. de/fileadmin/published_content/5.Qualitaet_und_Sicherheit/ PDF/2012_DGU_Whitebook_Medical_Care_2ndEdition.pdf.

20. Information. TKmed website. https://www.tkmed-direkt.org/ information/.

21. Ernstberger, Schmucker: Das Projekt TeleQualy, in: Orthopaedie und Unfallchirurgie Mitteilungen und Nachrichten (OUMN) 10/2014, S. 495-496. doi:10.1055/s-0034-1394208.

22. Nerlich M, Balas EA, Schall T, et al. Teleconsultation practice guidelines: report from G8 Global Health Applications Subproject 4. Telemed J E Health. 2002;8(4):411-418. doi:10.1089/15305620260507549.

23. Nerlich M, Mohr MT, Rdal DG. The ups and downs of the International Society for Telemedicine. J Telemed Telecare. 2004;10 Suppl 1:76-78. doi:10.1258/1357633042614311.

24. Eberl R, Biskup K, Reckwitz N, Muhr G, Clasbrummel B. [The televisit system in patients care after discharge in clinical use-first experiences]. Biomed Tech (Berl). 2005;50(5):132-136. doi:10.1515/bmt.2005.019.

25. Guerdal D, Leis A, Chouvarda I, Lekka I, Nerlich M, Maglaveras N: A Home Care System used in post-operative patient management. E-Health, Proceedings of Med-e-Tel 2006, The International Trade Event and Conference for eHealth, Telemedicine and Health ICT. p. 59. https://www.isfteh.org/files/media/eHealth_book_2006_1. pdf.

26. TeleArzt website. https://www.tele-arzt.com/.

27. Videosprechstunde: telemedizinisch gestützte Betreuung von Patienten. https://www.kbv.de/html/videosprechstunde.php.

28. Fernbehandlung: Weg frei für die Telemedizin. https://www. aerzteblatt.de/archiv/198076/Fernbehandlung-Weg-frei-fuer-dieTelemedizin.

29. Telemedizin. http://www.bundesaerztekammer.de/aerzte/ telematiktelemedizin/telemedizin/.

30. Bayern für mehr Förderung der Telemedizin. https://www. aerztezeitung.de/praxis_wirtschaft/e-health/telemedizin/ article/858417/regelversorgung-bayern-foerderung-telemedizin. html.

31. General Data Protection Regulation. https://dsgvo-gesetz.de/.

32. Forschung. TraumaRegister website. http://www.traumaregisterdgu.de/de/forschung.html. 